\title{
REAL REPRESENTATIONS OF METACYCLIC GROUPS
}

\author{
LARRY C. GROVE
}

Problem 14 in R. Brauer's survey article Representations of finite groups [1] asks for a characterization of the number of irreducible complex representations of a group $G$ that are equivalent to representations over the real field $R$. The question is answered here for a class of metacyclic groups, the answer being an arithmetic function of the parameters that appear in presentations of the groups.

Suppose $G$ is a metacyclic group having a cyclic normal subgroup $A=\langle a\rangle$ of order $m$ and cyclic quotient group $G / A=\langle b A\rangle$ of order $s$, with $b^{-1} a b=a^{r}$ and $b^{s}=a^{t}, 1 \leqq r \leqq m-1,0 \leqq t \leqq m-1$. Then by $[2$, p. 334], we have

$$
(m, r)=1, \quad t r \equiv t(\bmod m), \quad \text { and } \quad r^{r} \equiv 1(\bmod m) .
$$

For later use we set $d=\left(m, t, r^{s / 2}+1\right)$ when $s$ is even.

If $\zeta \in C$ is a primitive $m$ th root of unity then all irreducible complex characters of $A$ are obtained by setting $\phi_{i}(a)=\zeta^{i}, i=0, \cdots, m-1$. Each $\phi_{i}$ gives rise to an induced character $\theta_{i}=\phi_{i}{ }^{G}$ of $G$, and we wish to investigate which of the irreducible characters among the $\theta_{i}$ are characters of representations over $R$. The induced character $\theta_{i}$ is defined by the formula

$$
\begin{aligned}
\theta_{i}(x) & =(1 / m) \sum_{i \in G} \phi_{i}\left(t^{-1} x t\right) \quad \text { if } x \in A, \\
& =0 \quad \text { if } x \notin A .
\end{aligned}
$$

Let us compute $\theta_{i}$ more explicitly. We have in general $b^{-i} a b^{i}=a^{r^{j}}$, and so

$$
\begin{aligned}
\theta_{i}\left(a^{n}\right) & =(1 / m) \sum_{j=0}^{s-1} \sum_{k=0}^{m-1} \phi_{i}\left(a^{-k} b^{-j} a^{n} b^{j} a^{k}\right) \\
& =\sum_{j=0}^{s-1} \phi_{i}\left(b^{-j} a b^{j}\right)^{n}=\sum_{j=0}^{s-1} \zeta^{i r^{j} n} .
\end{aligned}
$$

For each $\theta_{i}$ set $\nu\left(\theta_{i}\right)=(1 / m s) \sum_{x \in G} \theta_{i}\left(x^{2}\right)$. If $\theta_{i}$ is irreducible then $\nu\left(\theta_{i}\right)=1$ if and only if $\theta_{i}$ is the character of a real representation, by the Theorem of Frobenius and Schur [3, p. 22].

Proposition 1. Suppose $s$ is odd. Then $\nu\left(\theta_{i}\right)=0$ for every irreducible $\theta_{i}$, and so no irreducible $\theta_{i}$ is the character of a real representation.

Received by the editors July 15, 1968. 
Proof. We have $\left(b^{k} a^{n}\right)^{2}=b^{2 k} a^{n\left(r^{k}+1\right)}$, so $\left(b^{k} a^{n}\right)^{2} \in A$ if and only if $s \mid k$, since $s$ is odd. We may assume $0 \leqq k \leqq s-1$ and still obtain all elements of $G$ in the form $b^{k} a^{n}$, so $\left(b^{k} a^{n}\right)^{2} \in A$ if and only if $k=0$. Thus

$$
\begin{aligned}
\nu\left(\theta_{i}\right) & =(1 / m s) \sum_{k=0}^{s-1} \sum_{n=0}^{m-1} \theta_{i}\left(b^{2 k} a^{n(r k+1)}\right) \\
& =(1 / m s) \sum_{n=0}^{m-1} \theta_{i}\left(a^{2 n}\right)=(1 / m s) \sum_{n=0}^{m-1} \sum_{j=0}^{s-1} \zeta^{2 i n r} \\
& =(1 / m s) \sum_{j} \sum_{n}\left(\zeta^{2 i r^{j}}\right)^{n} .
\end{aligned}
$$

If $m \nmid 2 i$, then $m\left\{2 i r^{i}\right.$ since $(m, r)=1$, and then $\sum_{n}\left(\zeta^{2 i r^{j}}\right)^{n}$ $=\left(1-\zeta^{2 i r^{i} m}\right) /\left(1-\zeta^{2 i r^{i}}\right)=0$, and so $\nu\left(\theta_{i}\right)=0$. If $m \mid 2 i$ then either $i=0$ or $i=m / 2$, with $m$ even. In either case each summand is 1 , so $\nu\left(\theta_{i}\right)=1$. Observe, however, that if $m$ is even then $r-1$ is even, and we have $m \mid(m / 2)(r-1)$. Thus $\theta_{i}$ is reducible if $i=m / 2$ (see [2, p. 335]). Likewise $\theta_{i}$ is reducible if $i=0$, and the proof is complete.

In view of Proposition 1 we suppose from this point on that s is even.

THEOREM 1. If $\theta_{i}$ is irreducible, then $\nu\left(\theta_{i}\right)=1$ if and only if $i \equiv$ $0(\bmod m / d)$.

Proof. We have $\theta_{i}\left(\left(b^{k} a^{n}\right)^{2}\right)=\theta_{i}\left(b^{2 k} a^{n\left(r^{k}+1\right)}\right)=0$ unless $k=0$ or $k=s / 2$. Thus

$$
\begin{aligned}
\nu\left(\theta_{i}\right) & =(1 / m s) \sum_{n=0}^{m-1}\left[\theta_{i}\left(a^{2 n}\right)+\theta_{i}\left(a^{t+n\left(r^{\circ / 2}+1\right)}\right)\right] \\
& =(1 / m s) \sum_{n}\left[\sum_{j=0}^{s-1} \zeta^{2 i n r^{i}}+\sum_{j=0}^{s-1} \zeta^{i n r^{3}\left(r^{8 / 2}+1\right)+i t r^{j}}\right] .
\end{aligned}
$$

Since $t r \equiv t(\bmod m)$ we have $t r^{i} \equiv t(\bmod m)$, and so $\zeta^{i t r^{i}}=\zeta^{i t}$. Observe that

$$
\begin{aligned}
\sum_{n=0}^{m-1}\left(\zeta^{2 i r^{j}}\right)^{n} & =m & & \text { if } m \mid 2 i, \\
& =0 & & \text { if } m \nmid 2 i,
\end{aligned}
$$

and that

$$
\sum_{n=0}^{m-1} \zeta^{i t\left(\zeta^{i r^{j}\left(r^{\prime / 2}+1\right)}\right)^{n}=m \zeta^{i t}} \quad \text { if } m \mid i\left(r^{8 / 2}+1\right),
$$

Thus $\nu\left(\theta_{i}\right)=B+D \zeta^{i t}$, where 


$$
\begin{aligned}
B & =1 & \text { if } m \mid 2 i, & D & =1 & \text { if } m \mid i\left(\boldsymbol{r}^{/ / 2}+1\right), \\
& =0 & \text { if } m \nmid 2 i, & & =0 & \text { if } m \nmid i\left(\boldsymbol{r}^{/ / 2}+1\right) .
\end{aligned}
$$

But $m \mid 2 i$ only if $i=m / 2$, with $m$ even, and in that case $\theta_{i}$ is reducible, as shown above. Thus $B=0$ and $\nu\left(\theta_{i}\right)=1$ if and only if $D=\zeta^{i t}=1$, i.e. if and only if $m \mid i\left(r^{8 / 2}+1\right)$ and $m \mid i t$.

In other words, we want all common solutions to the pair of congruences $i\left(r^{s / 2}+1\right) \equiv 0(\bmod m), i t \equiv 0(\bmod m)$ in the range $1 \leqq i \leqq m$ -1 . Since $i t \equiv 0(\bmod m)$ if and only if $i \equiv 0(\bmod m /(m, t))$, and $i\left(r^{s / 2}+1\right) \equiv 0(\bmod m)$ if and only if $i \equiv 0\left(\bmod m /\left(m, r^{s / 2}+1\right)\right)$, we have a common solution if and only if

$$
i \equiv 0\left(\bmod \left[m /(m, t), m /\left(m, r^{s / 2}+1\right)\right]\right) .
$$

But $\left[m /(m, t), m /\left(m, r^{s / 2}+1\right)\right]=m / d$, so we have shown that $\nu\left(\theta_{i}\right)=1$ if and only if $i \equiv 0(\bmod m / d)$.

Let us restate the original question in the light of Theorem 1. For each $k, 1 \leqq k \leqq d-1$, set $\psi_{k}=\theta_{k m / d}$. We wish to determine which of the characters $\psi_{k}$ are irreducible and which ones are equal to one another, and then to count the distinct irreducible $\psi_{k}$.

Proposition 2. The character $\psi_{k}$ is reducible if and only if $\mathrm{kr}^{j}$ $\equiv k(\bmod d)$ for some $j, 1 \leqq j \leqq s-1$.

Proof. It is shown in $[2$, p. 335$]$ that $\psi_{k}$ is reducible if and only if $m \mid k m\left(r^{j}-1\right) / d$ for some such $j$, and that is equivalent with the stated proposition.

Similarly, we have

Proposition 3. $\psi_{k}=\psi_{n}$ if and only if $k r^{i} \equiv n(\bmod d)$ for some $j, 1 \leqq j$ $\leqq s-1$.

Those metacyclic groups all of whose irreducible characters are either one dimensional or else among the characters $\theta_{i}$ are characterized in [2, p. 336]. We are now in a position to answer Brauer's question for such groups.

TheOREM 2. Suppose that $G$ is metacyclic and that all nonlinear irreducible characters of $G$ are induced from the cyclic subgroup $A$. If $s$ is even then $G$ has exactly $(d-(d, r-1)) / s$ inequivalent absolutely irreducible nonlinear representations over the real field $R$. If $s$ is odd, then $G$ has no absolutely irreducible nonlinear representations over $\boldsymbol{R}$.

Proof. The case where $s$ is odd is covered by Proposition 1. Suppose then that $s$ is even. By $\left[2\right.$, p. 336] we know that if $r^{i} i \equiv i(\bmod m)$, 
with $1 \leqq j \leqq s-1$, then $r i \equiv i(\bmod m)$. Set $Y=\{1,2, \cdots, d\}$, and define $\beta: Y \rightarrow Y$ by setting $\beta(k)=r k(\bmod d)$. Since $(r, d)=1, \beta$ is a permutation of $Y$. Since $r^{s} \equiv 1(\bmod d)$ the order of $\beta$ divides $s$. Note that if $\beta^{i}(k)=r^{i} k=k$ in the integers $\bmod d$, then in fact $\beta(k)=k$, if $j \leqq s-1$. It follows that the orbits of $\beta$ in $Y$ have either one or $s$ elements. It is clear from Propositions 2 and 3 that $\psi_{k}$ is reducible if and only if $\{k\}$ is a one-element orbit, and that $\psi_{k}=\psi_{n}$ if and only if $k$ and $n$ are in the same orbit. But $\{k\}$ is an orbit if and only if $d \mid k(r-1)$. i.e. if and only if

$$
k=d /(d, r-1), 2 d /(d, r-1), \cdots, d=(d, r-1) d /(d, r-1) .
$$

Thus the number of $s$-element orbits, and hence the number of $\psi_{k}$ that are characters of real representations, is $(d-(d, r-1)) / s$.

REMARK. Note that $r^{n}+1=(r-1) \sum_{k=1}^{n-1} r^{k}+(r+1)$. Thus for $n \geqq 1$ we have

$$
\begin{array}{rlrl}
\left(r-1, r^{n}+1\right)=(r-1, r+1) & =1 & & \text { if } r \text { is even, } \\
& =2 & \text { if } r \text { is odd. }
\end{array}
$$

As a result $(d, r-1)=(m, t, r+1, r-1)=2$ if $r$ is odd and $m$ and $t$ are both even, and $(d, r-1)=1$ in all other cases.

Let us consider Theorem 2 for two particular examples.

1. The dihedral group $D_{m}$ of order $2 m$ is metacyclic, with $s=2$, $t=0$, and $r=m-1$. Thus $d=(m, m)=m$, and $(d, r-1)=2$ if $m$ is even, $=1$ if $m$ is odd. We conclude that $D_{m}$ has $(m-2) / 2$ nonlinear absolutely irreducible real representations if $m$ is even, and $(m-1) / 2$ if $m$ is odd.

2. The generalized quaternion group $Q_{n}$ of order $4 n$ is metacyclic, with $m=2 n, s=2, t=n$, and $r=2 n-1$. Then $d=(2 n, n, 2 n)=n$, and $(d, r-1)=1$ if $n$ is odd, $=2$ if $n$ is even. Thus $Q_{n}$ has $(n-1) / 2$ nonlinear absolutely irreducible real representations if $n$ is odd, and $(n-2) / 2$ if $n$ is even.

Corollary to Theorem 2. Suppose $G$ is a finite nonabelian group having a cyclic subgroup of index 2 . Then all nonlinear irreducible complex representations of $G$ are equivalent with real representations if and only if $G$ is a dihedral group $D_{m}$.

The proof will be omitted. The referee has pointed out that the conclusion of the corollary holds under the hypotheses that $[G:\langle a\rangle]$ $=2$ and $G$ has an absolutely irreducible faithful nonlinear representation over $R$.

I wish to thank C. T. Benson and C. W. Curtis for a number of fruitful conversations regarding this material. 


\section{REFERENCES}

1. R. Brauer, "Representations of finite groups" in Lectures on modern mathematics, edited by T. L. Saaty, Wiley, New York, 1963.

2. C. Curtis and I. Reiner, Representation theory of finite groups and associative algebras, Interscience, New York, 1962.

3. W. Feit, Characters of finite groups, Lecture Notes, Yale University, New Haven, Conn., 1965.

UNIVERSITY OF OREGON 